\title{
La división digital en el proceso de integración de las NTIC en la educación. Diferencias de género entre alumnos de E.S.O. de la comunidad valenciana.[1]
}

\author{
Bernardo Gargallo López bernardo.gargallo@uv.es \\ Jesús Suárez Rodríguez Jesús.M.Rodriguez@uv.es \\ $M^{a}$. Consuelo Belloch Ortí Consuelo.Belloch@uv.es \\ Universidad de Valencia
}

\section{Introducción.}

La "división digital" (también traducida del inglés "digital divide" como "divisoria digital" y "brecha digital") es un concepto acuñado en los últimos años como expresión de diferentes aspectos negativos de la introducción de las TIC y de su impacto en los distintos ámbitos de la sociedad. De hecho, se habla de brecha digital en el sentido más coloquial aludiendo a diferencias sustantivas entre países ricos y países pobres en el acceso a la red (infraestructuras, accesibilidad...) -hay zonas del mundo prácticamente "desconectadas", "en sombra"-. Es claro que la información es elemento sustantivo para el desarrollo económico y, en un contexto de globalización, de la que los más optimistas esperaban la reducción de las diferencias y la redistribución de la riqueza, las distancias se pueden incrementar en vez de reducir "globalización" de la pobreza para gran parte del mundo- si no se toman medidas para evitarlo de modo que el acceso a los recursos tecnológicos necesarios se convierta en una prioridad política internacional.

Se trata de un tema candente que ha ido recibiendo atención creciente tanto desde las políticas públicas como desde los estudios y análisis académicos (Marina, 1999; Rhode y Shapiro, 2000; Castells, 2000; Grupo Web de MS, 2001; Kerrey, 2000; Shashaani y Khalili, 2001).

El problema, sin embargo, presenta múltiples facetas. Así, las diferencias entre géneros constituyen uno de los núcleos que integran esta problemática -hay páginas web dedicadas exclusivamente al tema que aquí nos ocupa, como http://www.worldbank. org/gender/digitaldivide/, que incluye abundante documentación, junto con otros como la edad, la capacidad económica (pobres-ricos), la ubicación espacial en determinadas zonas de los países (rural-urbana), o en una zona determinada del mundo (Norte-Sur), etc. Sin embargo, la mayor parte de los estudios y propuestas se refieren a la población general y se concentran en los aspectos básicos y contextuales de los procesos de integración de las TIC infraestructuras y utilización- (Price, 2000; Singleton y Mast, 2000; OCDE, 2001).

El trabajo que presentamos aquí pretende analizar las diferencias de género en el colectivo de alumnos de Educación Secundaria Obligatoria de la Comunidad Valenciana, incluyendo infraestructuras y accesibilidad, utilización, formación, actitudes, y valoración del uso de Internet en la calidad de la educación.

\section{La investigación.}

Este estudio parte de una investigación[2] más amplia que pretendía valorar la integración de Internet en los centros escolares de la Comunidad Valenciana (Gargallo, Suárez, Morant, Marín, Martínez y Díaz, 1991).

El diseño del trabajo integraba una parcela cuantitativa, con un diseño de encuesta que recogía información, a través de cuestionarios on-line, de diferentes agentes claves para el propósito central (directores, coordinadores de informática, profesores y alumnos), haciendo uso de triangulación de perspectivas, al cruzar la información proporcionada por las cuatro fuentes, y una parcela cualitativa, con estudio de casos (visitas a centros con experiencias significativas), con lo que se hacía también uso de 
triangulación metodológica.

El procedimiento de muestreo de la parcela cuantitativa del estudio, la que aquí nos interesa, fue aleatorio estratificado en función de la provincia (Valencia, Castellón, Alicante), tipo de centro (público-no público), y curso (primero-cuarto), logrando para los alumnos, parte de cuyos resultados analizamos aquí, una excelente representatividad, con un nivel de confianza del 95\% y un error máximo del 1,5\% para el conjunto de la Comunidad Valenciana. La muestra de alumnos estaba integrada por 2311 sujetos (388 de $1^{\circ}$ de ESO, 450 de $2^{\circ}, 806$ de $3^{\circ}$ y 667 de $4^{\circ}$ ), 1130 chicos y 1165 chicas (16 no contestaronal ítem correspondiente al género). En cualquier caso, se ha llevado a cabo una ponderación respecto a las variables de estratificación del conjunto de la muestra en todos los análisis realizados.

\section{Los resultados y su discusión.}

Analizamos a continuación, brevemente, las diferencias observadas en cuatro bloques fundamentales de los que obtuvimos información a través de los cuestionarios antes aludidos: conexión y uso, formación, actitudes, y valoración de las repercusiones del uso de Internet en la calidad de la educación. Las diferencias se han analizado en cada uno de estos bloques temáticos haciendo uso de metodología univariada (ANOVA) y multivariada (análisis discriminante) como perspectivas complementarias. Se ha realizado también un análisis discriminante conjunto para las distintas variables significativas de los cuatro bloques, lo que permitirá llevar a cabo una reintegración final y una caracterización de los dos géneros en lo referente a elementos significativos relativos a las diferencias existentes entre ambos.

El análisis discriminante es el procedimiento adecuado si se quiere profundizar en las interpretación de las diferencias que detecta el análisis univariado y multivariado. Las situaciones valoradas se basan en un modelo MANOVA simple, con una variable Independiente de tipo entre (género en este caso) y múltiples variables dependientes. El análisis discriminante, en principio, es una de los procedimientos más adecuados para identificar las relaciones causales potenciales respecto a las variables diferenciales y ofrecer una interpretación adecuada de las mismas. Este procedimiento permite no solamente verificar las diferencias entre los perfiles multivariados de los diferentes grupos que se comparan sino, también, establecer dimensiones que sintetizan estas diferencias. Estas dimensiones, similares a los factores del análisis factorial, simplifican la situación a un menor número de indicadores -la reducen-, y adquieren sentido en base a las relaciones que presentan con las variables dependientes originales. Asimismo, el análisis discriminante es útil para situaciones en las que se desea construir un modelo de pronóstico de pertenencia al grupo basándose en las características observadas para cada caso. El procedimiento genera una función discriminante (o, para más de dos grupos, un conjunto de funciones discriminantes) basándose en las combinaciones lineales de las variables predictoras que proporcionan las mayor discriminación entre los grupos (Hair, Anderson, Tatham y Black, 1999). El método seguido ha sido identificar a través de pruebas de significación univariadas y del correspondiente modelo multivariado -utilizando el procedimiento de paso-a-paso-, las variables que establecen diferencias significativas entre los grupos y llevar a cabo la solución completa del discriminante basada únicamente en dichas variables. De esta manera se ha utilizado el procedimiento según una aproximación de construcción de modelo, ya que no es ésta una situación en la que exista un modelo -o varios- inicialmente propuesto, y se trata de indagar sistemáticamente para encontrar la mejor representación de las informaciones obtenidas. Con ello se ha pretendido evitar los riesgos que apuntan diferentes autores (Stevens, 1996) respecto a la utilización "automática" de procedimientos de análisis de datos, especialmente cuando los conocimientos asentados sobre una temática aconsejan incidir en una perspectiva exploratoria.

\subsection{Conexión y uso}

\subsubsection{Resultados del análisis de varianza (ANOVA)[3]}

Las medias son superiores en los chicos en 44 de las 47 variables analizadas (Tabla 1)[4] y las diferencias son significativas en más de la mitad de las mismas. De ellas algunas son especialmente importantes: así, la disponibilidad de conexión a Internet en casa (ítem 1), las horas semanales de conexión en casa (ítem 2), las horas semanales dedicadas en el centro en horario escolar a actividades de las asignaturas (ítem 5) o a actividades curriculares fuera del horario de clase (ítem 6), las horas dedicadas a formación en informática en actividades extraescolares (ítem 7). Igualmente, el uso de Internet para realizar actividades con compañeros y profesores (ítem 10), su utilización para obtener información (ítems 11 y 12). Por lo 
que respecta a los servicios de Internet (ítems 14-20), tanto en los más usuales -correo electrónico o chats, como en los más especializados -FTP, foros, Telnet o diseño de páginas web-, siempre la media de uso en el centro es superior en los chicos, siendo la diferencia significativa en la mayoría de los ítems.

Más sorprendente es la diferencia existente en el uso en las asignaturas en el centro (ítems 21-37), dado que se trata de los mismos centros, en que también las medias son superiores en los chicos, si exceptuamos el caso de Lengua Castellana, siendo casi en la mitad de los casos las diferencias significativas. Es lógico pensar, en este caso, que es este resultado es una síntesis de percepciones y realidades objetivas difícil de desentrañar en este momento.

También en los usos fuera del centro (ítems 40-47) son superiores las medias en los chicos, excepto en los chats en que ocurre al revés, siendo las diferencias significativas en el uso de otro ordenador (Telnet), FTP, diseño de páginas web y otros. En último término, los chicos son más capaces de llevar a cabo usos especializados de Internet que las chicas.

Las diferencias significativas a favor de los varones también se dan en actividades no vinculadas al aprendizaje referido al currículo: así, el uso del ordenador para jugar (ítem 3), o lashoras dedicadas a jugar en línea (ítem 9), lo que probablemente tiene que ver con las preferencias lúdicas de unos y otros.

En cualquier caso, es preciso tener presente que los niveles de utilización de Internet son, en la totalidad de las cuestiones consideradas, bajos o muy bajos en el conjunto de la población de alumnos de E.S.O. de la Comunidad Valenciana. Por tanto, sería esperable que al crecer los niveles de utilización estas diferencias se hicieran más ostensibles, extendiéndose a muchas otras cuestiones.

\section{Tabla 1.-Diferencias de uso entre chicos y chicas}

\begin{tabular}{|c|c|c|c|c|c|c|}
\hline İtems relativos a FORMACIÓN para el uso de Internet & $\begin{array}{l}\text { Media } \\
\text { Hombre }\end{array}$ & D. Típica & $\begin{array}{l}\text { Media } \\
\text { Mujer }\end{array}$ & D. Típica & F. Anova & $\begin{array}{l}\text { Signifi- } \\
\text { cación }\end{array}$ \\
\hline 1.-¿¿Cómo calificarías tu nivel de conocimiento y manejo de Internet? & 2,69 & 1,13 & 2,25 & ,93 & 98,19 &, 000 \\
\hline $\begin{array}{l}\text { 2.-He recibido formación acerca de Internet en el centro en la asignatura } \\
\text { de informática }\end{array}$ & 2,02 & 1,27 & 1,98 & 1,26 & ,36 & 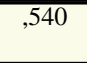 \\
\hline 3.-He recibido formación... en otras asignaturas. & 1,27 & ,65 & 1,22 & ,55 & 3,06 & ,080 \\
\hline 4.-He recibido formación... en alguna actividad extraescolar en el centro. & 1,45 & ,94 & 1,35 & ,83 & 6,60 & ,010 \\
\hline $\begin{array}{l}\text { 5.-He recibido formación... } \mathrm{n} \text { alguna entidad privada (academia, centro } \\
\text { de informática, etc). }\end{array}$ & 1,56 & 1,10 & 1,51 & 1,08 & ,83 & ,359 \\
\hline 6.-He recibido formación....con la ayuda de compañeros o amigos. & 2,51 & 1,34 & 2,47 & 1,27 & 471 & ,493 \\
\hline 7.-He recibido formación... en la familia & 2,10 & 1,38 & 2,21 & 1,41 & 3,23 & ,072 \\
\hline 8.-He recibido formación... a través de otros (especificar) & 1,87 & 1,48 & 1,53 & 1,19 & 24,67 & ,000 \\
\hline 9.-Mi formación con relación a Internet ha consistido en... & 1,44 & ,87 & 1,32 & ,66 & 14,06 & ,000 \\
\hline $\begin{array}{l}\text { 10.-Donde mejor se aprende el uso de Internet es en el centro en la } \\
\text { asignatura de Informática. }\end{array}$ & 2.78 & 1,36 & 2,93 & 1,31 & 6,45 & ,011 \\
\hline 11.-Se aprende mejor... en el centro en otras asignaturas. & 1,57 & ,89 & 1,68 & ,93 & 7,41 & ,007 \\
\hline 12.-Se aprende mejor... en alguna actividad extraescolar en el centro. & 2,12 & 1,27 & 2,37 & 1,25 & 19,74 & ,000 \\
\hline $\begin{array}{l}\text { 13.-Se aprende mejor... en alguna entidad privada (academia, centro de } \\
\text { informática, etc). }\end{array}$ & 3,32 & 1,55 & 3,66 & 1,46 & 27,72 & ,000 \\
\hline 14.-Se aprende mejor con la ayuda de compañeros o amigos. & 2,94 & 1,26 & 2,96 & 1,15 & 12 & ,726 \\
\hline 15.-Se aprende mejor...en la familia & 2,39 & 1,33 & 2,61 & 1,30 & 3,23 & ,072 \\
\hline 16.-Se aprende mejor en otros (especificar) Respuesta (C18g). & 1,80 & 1,39 & 1,46 & 1,06 & 24,52 & ,000 \\
\hline 17.- Formación recibida en el manejo del correo electrónico? & 2,19 & 1,34 & 2,16 & 1,32 & 224 & ,636 \\
\hline 18.- Formación recibida en el Manejo de buscadores? & 2,63 & 1,41 & 2,39 & 1,32 & 16,70 &, 000 \\
\hline 19.-Formación recibida en el manejo de bases de datos? & 2,11 & 1,22 & 1,97 & 1,11 & 7,21 & ,007 \\
\hline 20.-Formación recibida en transferencia de ficheros (FTP)? & 1,83 & 1,20 & 1,50 & ,99 & 25,28 &, 000 \\
\hline 21.-Formación recibida en Charlas interactivas? & $\overline{2,53}$ & 1,50 & $\overline{2,58}$ & $\overline{1,53}$ & 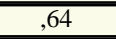 & 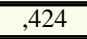 \\
\hline 22.-Formación recibida en foros de discusión? & 1,79 & 1,19 & 1,78 & 1,17 &, 065 & ,799 \\
\hline 23.-Formación recibida en el uso de otro ordenador (Telnet)? & $\overline{1,70}$ & $\overline{1,17}$ & $\overline{1,57}$ & $\overline{1,00}$ & $\overline{7,47}$ & ,006 \\
\hline 24.-Formación recibida en el diseño de páginas web? & 1.64 & 1.13 & 1,54 & ,96 & 5,26 & 022 \\
\hline 25.-Formación recibida en la utilización escolar de Internet? & 2,25 & 1,36 & 2,21 & 1,31 & ,431 & ,512 \\
\hline $\begin{array}{l}\text { 26.-Formación recibida en el comportamiento correcto en la red } \\
\text { (ciberética)? }\end{array}$ & 2,22 & 1,41 & 2,18 & 1,39 & ,517 & ,472 \\
\hline
\end{tabular}

\subsubsection{Resultados del análisis discriminante}

Los resultados obtenidos en la prueba $\mathrm{M}$ de Box nos llevan a realizar la interpretación de los resultados obtenidos en el discriminante con precaución, dado que se obtiene un valor significativo ( $<<0.000)$, esto es, no se cumple el supuesto de homogeneidad de la varianza en las poblaciones analizadas. Dado que el análisis discriminante se realiza sobre dos grupos poblacionales, obtenemos una única función discriminante. Dicha función presenta una correlación canónica moderada (.344) y un alto nivel de significación (.000). En la tabla siguiente presentamos los coeficientes estandarizados y la matriz de 
estructura de la función canónica resultante.

Tabla 2.-Puntuaciones en la matriz de estructura y coeficientes estandarizados

\begin{tabular}{|l|c|c|}
\hline & $\begin{array}{c}\text { Matriz de } \\
\text { estructura (a) }\end{array}$ & $\begin{array}{c}\text { Coeficientes } \\
\text { estandarizados }\end{array}$ \\
\hline Horas semanales dedicadas en casa a usar el ordenador para "jugar". &, 765 &, 927 \\
\hline Frecuencia de uso fuera del centro para diseño de páginas web. &, 387 &, 283 \\
\hline Frecuencia de uso de Internet fuera del centro para otras actividades. &, 332 &, 224 \\
\hline Horas semanales dedicadas en casa a estar conectado. &, 311 &, 320 \\
\hline Frecuencia de uso fuera del centro para charlas (chats, IRC, etc.). & -.018 &,- 353 \\
\hline Horas semanales dedicadas en casa a usar el ordenador para otras cosas -sin jugar- & -.001 &,- 568 \\
\hline
\end{tabular}

(a) Correlaciones irtragrupo combinadas ertre las variables discrininantes y las funciomes discriminantes canónicas tipificadas. Variables ordenadas por el tamaño de la oorelación con la fimcín.

La función discriminante obtenida recoge principalmente ítems relacionados con la frecuencia de uso del ordenador e Internet fuera del centro escolar, presentando una configuración bipolar. En el polo positivo, el ítem que más aportación realiza a la función es "usar el ordenador para jugar" (,927), junto a otros que presentan menor aportación (horas de conexión, diseño de páginas web y uso de Internet para otras actividades). En el polo negativo el ítem que realiza una mayor aportación a la función es "usar el ordenador para otras cosas -sin jugar-" (-,568), contribuyendo en menor medida el uso para charlas enlínea.

Tabla 3.-Funciones en los centroides de los grupos

\begin{tabular}{|l|c|}
\hline \multicolumn{2}{|c|}{ Función } \\
\hline Chico &, 376 \\
Chica &,- 356 \\
\hline
\end{tabular}

Por otro lado, los resultados de la función discriminante obtenida en los centroides de los grupos (ver tabla 3) evidencian que los chicos se sitúan en el polo positivo de la función (,376) y las chicas en el negativo (,356), lo que significa que los chicos se caracterizan por utilizar en mayor medida el ordenador e Internet para jugar, y para diseñar páginas web o realizar otras actividades no relacionadas con sus estudios, mientras que las chicas utilizarían en menor medida Internet para otras actividades no lúdicas[5] y, especialmente para favorecer la comunicación informal con -participando en charlas en-línea-. No obstante, hay que tener presente que los valores obtenidos en las funciones de los centroides de ambos grupos indican que existe un cierto solapamiento entre ambos grupos.

\section{Tabla 4.- Resultados de la clasificación}

\begin{tabular}{|ll|c|c|r|}
\cline { 2 - 3 } \multicolumn{1}{c|}{} & \multicolumn{2}{c|}{$\begin{array}{c}\text { Grupo de pertenencia } \\
\text { pronosticado }\end{array}$} & \multirow{2}{*}{ Total } \\
\hline & Género & Chico & Chica & \\
\hline Original Recuento & Chico & 616 & 514 & 1130 \\
& Chica & 387 & 778 & 1165 \\
& Casos desagrupados & 11 & 5 & 16 \\
\hline \multicolumn{2}{c|}{ \% Chico } & 54,5 & 45,5 & 100,0 \\
& Chica & 33,2 & 66,8 & 100,0 \\
& Casos desagrupados & 66,6 & 33,4 & 100,0 \\
\hline \multicolumn{2}{|c|}{ Clasificados correctarnente el $60,7 \%$ de los casos agrupados originales. } \\
\hline
\end{tabular}

Estos resultados se constatan en la tabla presentada sobre los resultados de la clasificación de los alumnos atendiendo a su género. En ella observamos que únicamente el grupo de las chicas alcanza un porcentaje de clasificación correcta (66,8\%) adecuado, mientras que los chicos no son bien clasificados atendiendo a los resultados de la función canónica, dado que el nivel de clasificación correcta (54,5\%) se sitúa en torno al $50 \%$.

Atendiendo a que no se cumple el supuesto de homogeneidad de las varianzas y a que los resultados obtenidos en la clasificación de los sujetos por la función discriminante canónica no presentan altos 
porcentajes de clasificación correcta en ambos grupos, únicamente podemos concluir que se apunta una tendencia de los chicos a utilizar en mayor medida Internet como recurso para jugar, mientras que las chicas suelen utilizarlo con fines de comunicación y presentan un menor nivel de utilización -con cualquier fin- en su domicilio.

Como comentario final, hacemos constar que los datos del análisis univariado y multivariado de diferencias parecen traducir una mayor incorporación de los chicos al uso de Internet y una cierta divisoria digital cuyas causas habría que analizar y que habría que atajar cuanto antes[6]. En todo caso, se trata de una realidad con la que hay que contar a la hora de diseñar planes de dotación y de integración en los centros escolares y que tanto los administradores como los directores, los coordinadores de informática y los profesores deben tener presente.

\subsection{Formación}

\subsubsection{Resultados del análisis de varianza}

Los datos se recogen en la Tabla 5[7]. Los varones se sienten más capacitados que las mujeres (ítem 1), y también han recibido más formación reglada (ítem 9), en ambos casos con diferencia significativa de medias a su favor. A la hora de precisar dónde se ha recibido formación (ítems 2-8), también las medias son superiores en los chicos, excepto en el ítem 7 (formación recibida en la familia), aunque la diferencia sólo es significativa en dos ítems (ítem 4: formación recibida en alguna actividad extracurricular en el centro; e ítem 8: formación recibida a través de otros...).

Cuando se trata de valorar las modalidades de formación (ítems 10-16), las medias son superiores en las mujeres con la única excepción del ítem 16 ("se aprende mejor en otros..."), dándose diferencia significativa en cinco de los siete ítems, lo que significa que las chicas valoran más que los chicos las diferentes modalidades de formación a excepción de la última, en que ocurre lo contrario.

Sin embargo, a la hora de valorar la formación recibida en los diferentes contenidos de Internet (ítems 1726), las medias son superiores en los chicos, con la excepción de las charlas interactivas (ítem 21), lo que supone un mayor grado de satisfacción con la formación recibida en dichos contenidos. Además, la diferencia es significativa en cinco de los diez ítems (18: manejo de buscadores; 19: manejo de bases de datos; 20: transferencia de ficheros; 23: uso de otro ordenador, y 24: diseño de páginas web), que se refieren todos ellos a uso de servicios especializados de Internet.

Tabla 5.-Diferencias de formación entre chicos y chicas 


\begin{tabular}{|c|c|c|c|c|c|c|}
\hline 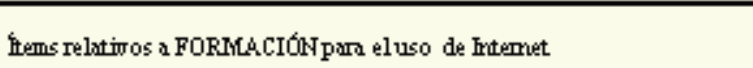 & $\begin{array}{c}\text { Medin } \\
\text { Hombre }\end{array}$ & $\begin{array}{c}\text { D. } \\
\text { T'́pica }\end{array}$ & $\begin{array}{l}\text { Mredin } \\
\text { Ifujer }\end{array}$ & $\begin{array}{c}\text { D. } \\
\text { Tímica }\end{array}$ & $\begin{array}{c}\text { F. } \\
\text { Anowa }\end{array}$ & $\begin{array}{l}\text { Signifi- } \\
\text { cación }\end{array}$ \\
\hline 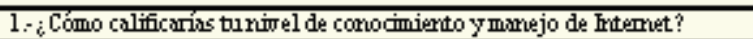 & 2,69 & 1,13 & 2,25 & 93 & 98,19 & 000 \\
\hline $\begin{array}{l}\text { 2. He recibido form ación acerca de frotennet en el certro en la asignatarna de } \\
\text { informática. }\end{array}$ & 2,02 & 1,27 & 198 & 1,26 & 36 & 540 \\
\hline 3. He recibido form ación.... en otras asignaturas. & 1,27 & 65 & 1,22 & 55 & 3,06 & 080 \\
\hline 4.-He recibido form ación... en alguna actividad extraescolar en el certro. & 1,45 & 94 & 1,35 & 83 & 6,60 & 010 \\
\hline $\begin{array}{l}\text { 5. He recibido formación... n alguna entidad prir ada (academina, centro de } \\
\text { informática, etc). }\end{array}$ & 1,56 & 1,10 & 151 & 1,08 & 83 & 359 \\
\hline 6. He recibido form ación. ...con ln ayuda de compríneros o amizos. & 2,51 & 1,34 & 2,47 & 1,27 & 471 & 493 \\
\hline 7.He recibido form ación.... en la familin & 2,10 & 138 & 221 & 1,41 & 323 & 072 \\
\hline 8. He recibido formación. ... a tranés de otros (especificar) & 1,87 & 1,48 & 1,53 & 1,19 & 24,67 & 000 \\
\hline 9.-Míform ación con relación a Intennet ha corsistido en... & 1,44 & 87 & 1,32 & 66 & 1406 & 000 \\
\hline $\begin{array}{l}\text { 10.-Donde mejor se aprende eluso de frtemet es en el centro en la } \\
\text { asignantra de Informática. }\end{array}$ & 2.78 & 136 & 293 & 131 & 6,45 & 011 \\
\hline 11.-Se qurende mejor... en el certro en otras asigntarns. & 1,57 & 89 & 1,68 & 93 & 7,41 & 007 \\
\hline 12.Se arrende mejor... en alguma actio ind extrascolar en el centro. & 2,12 & 1,27 & 2,37 & 125 & 19,74 & 000 \\
\hline $\begin{array}{l}\text { 13. Se aprende mejor... en alguna entidad prir ada (academin, centro de } \\
\text { informática, etc). }\end{array}$ & 332 & 1,55 & 3,66 & 1,46 & 27,72 & 000 \\
\hline 14.Se surende mejor con la aruda de comporieros o mizos. & 294 & 1,26 & 296 & 1,15 &, 12 & 726 \\
\hline 15.Se mrende mejor...en lo familia & 239 & 133 & 2,61 & 130 & 323 & 072 \\
\hline 16.Se aprende mejor en otros (especificar) Respuesta (C18g). & 1,80 & 139 & 1,46 & 106 & 24,52 & 000 \\
\hline 17. Formaciónrecibida en el lmanejo del conre electrónico? & 2,19 & 1,34 & 2,16 & 132 & 224 & 636 \\
\hline 18. Formación recibida en el Mnruejo de buscadores? & 2,63 & 1,41 & 239 & 132 & 16,70 & 000 \\
\hline 19.Formación recibida en elmmejo de bases de datos? & 2,11 & 1,22 & 197 & 1,11 & 721 & 007 \\
\hline 20.-Formaciónrecibida en tronsferencin de ficheros (FIP)? & 1,83 & 120 & 1,50 & 99 & 25,28 & 000 \\
\hline 21.Fomaciónrecibida en Charlas irteractios? & 2,53 & 1,50 & 2,58 & 153 & 64 & .424 \\
\hline 22.-Fomación recibida en foros de discusín? & 1,79 & 1,19 & 1,78 & 1,17 & 065 & 799 \\
\hline 23.Formación recibida en eluso de otro ordenador (Tehret)? & 1,70 & 1,17 & 1,57 & 100 & 7,47 & 006 \\
\hline 24-Formación recibida en el disếto de píginas web? & 1.64 & 1.13 & 1,54 & 96 & 526 & 022 \\
\hline 25.Fomación recibida en la utilianción escolar de Internet? & 2,25 & 136 & 221 & 131 & 431 & 512 \\
\hline $\begin{array}{l}\text { 26. Fomación recibida en el comportsmiento correcto en la red } \\
\text { (ciberética)? }\end{array}$ & 2,22 & 1,41 & 2,18 & 139 & 517 & 472 \\
\hline
\end{tabular}

\subsubsection{Resultados del análisis discriminante}

Los resultados obtenidos en el análisis discriminante indican que no se cumple el supuesto de homogeneidad de las varianzas entre los grupos de chicos y chicas, puesto que la prueba $\mathrm{M}$ de Box resulta significativa $(\mathrm{p}<0.000)$, lo que nos llevará a analizar los resultados obtenidos en el análisis discriminante con cierta precaución.

La función discriminante obtenida presenta una correlación canónica moderada $($,295) y un altonivel de significación $(, 000)$ en la prueba Lambda de Wilks. A ella se vinculan 6 ítems relativos a la formación sobre Internet: la percepción que el propio alumno tiene sobre su formación, la formación que ha recibido en la familia o por otros medios no formales, la formación relativa a dos servicios de Internet (correo electrónico y transferencia de ficheros (FTP) y al comportamiento ético en la red.

Los coeficientes estandarizados de los ítems y la matriz de estructura de la función discriminante (ver tabla 6), nos presentan una función discriminante bipolar, con una mayor carga del polo positivo que del negativo. Al polo positivo se vinculan ítems relativos a la percepción que el estudiante tiene sobre su conocimiento y manejo de Internet (,859), la valoración de la formación recibida en FTP, y la valoración de medios no formales para el aprendizaje de Internet. Mientras que al polo negativo, mucho más débilmente definido, se vincula principalmente el ítem que hace referencia a la familia como mejor medio para el aprendizaje de Internet $(-, 376)$, junto a la menor valoración de la formación recibida sobre el manejo del correo electrónico y en el comportamiento ético en la red.

Tabla 6.-Puntuaciones en la matriz de estructura y coeficientes estandarizados.

\begin{tabular}{|l|c|c|}
\hline & $\begin{array}{c}\text { Matriz de } \\
\text { Estructura (a) }\end{array}$ & $\begin{array}{c}\text { Coeficientes } \\
\text { Estandarizados }\end{array}$ \\
\hline ¿Cómo calificarías tu nivel de conocimiento y manejo de Internet? &, 777 &, 859 \\
\hline ¿Cómo ha sido la formación recibida en transferencia de ficheros (FTP)? &, 489 &, 425 \\
\hline ¿Dónde crees que se aprende mejor el uso de Intemet? Otros &, 473 &, 311 \\
\hline ¿Dónde crees que se aprende mejor el uso de Internet? En la farnilia &,- 112 &,- 376 \\
\hline ¿Cómo ha sido la formación recibida en el manejo del correo electrónico? &, 108 &,- 320 \\
\hline $\begin{array}{l}\text { ¿Cómo ha sido la formación recibida en el comportarniento correcto en la red } \\
\text { (ciberética)? }\end{array}$ &, 104 &,- 285 \\
\hline
\end{tabular}

(a) Conelaciones irtragrupo combinadas entre las variables discriminantes y las fimciones discriminartes camónicas tịificadas. Variables ordenadas por el tamaño de la correlación con la fimcín. 
El análisis de los resultados obtenidos en la función discriminante para los centroides de los grupos nos permite comprobar que los chicos se sitúan en el polo positivo de la función $(, 289)$ y las chicas en el polo negativo $(-, 329)$, aunque el hecho de que los valores obtenidos son relativamente bajos evidencia un fuerte solapamiento entre los dos grupos.

\section{Tabla 7.- Funciones en los centroides de los grupos}

\begin{tabular}{|l|c|}
\hline \multicolumn{2}{|c|}{ Función } \\
\hline Chico &, 289 \\
Chica &,- 329 \\
\hline
\end{tabular}

Esta evidencia es constatada en la tabla de clasificación de los alumnos atendiendo al grupo del alumno y al grupo pronosticado según los resultados obtenidos en la función discriminante canónica. El grupo con un mayor porcentaje de clasificación correcta es el de las alumnas (65,1\%), obteniéndose un menor porcentaje en el caso de los alumnos (55,2\%), ligeramente superior a la probabilidad previa del grupo, que se sitúa en el $50 \%$.

\section{Tabla 8.-Resultados de la clasificación}

\begin{tabular}{|cl|c|c|r|}
\cline { 2 - 3 } \multicolumn{1}{c|}{} & \multicolumn{2}{c|}{$\begin{array}{c}\text { Grupo de pertenencia } \\
\text { pronosticado }\end{array}$} & \multirow{2}{*}{ Total } \\
\hline & Género & Chico & Chica & \\
\hline Original Recuento & Chico & 623 & 507 & 1130 \\
& Chica & 406 & 759 & 1165 \\
& Casos desagrupados & 10 & 6 & 16 \\
\hline$\%$ & Chico & 55,2 & 44,8 & 100,0 \\
& Chica & 34,9 & 65,1 & 100,0 \\
& Casos desagrupados & 65,7 & 34,3 & 100,0 \\
\hline \multicolumn{2}{|c|}{ Clasificados correctamente el $60,2 \%$ de los casos agrupados originales } \\
\hline
\end{tabular}

En conclusión, únicamente podemos apuntar una tendencia de los chicos a tener una percepción más positiva sobre sus conocimientos y manejo de Internet, una mayor valoración sobre la formación recibida en transferencia de ficheros y una percepción más positiva sobre otros medios de aprendizaje de Internet no formales, mientras que las alumnas de secundaria parecen mostrar una preferencia por el aprendizaje en el entorno familiar y una menor valoración de la formación recibida sobre el correo electrónico y sobre el comportamiento ético en la red. Hablamos de tendencia dado que los resultados obtenidos, tanto en la comprobación del supuesto de homogeneidad de las varianzas como en la clasificación de los alumnos atendiendo a la función discriminante obtenida apuntan hacia una dificultad para la discriminación de los alumnos en función de estas variables en relación a su género, esto es hacia un solapamiento entre ambos grupos.

Como comentario final, los resultados del análisis univariado y multivariado de diferencias traducen de nuevo una cierta divisoria digital, con un perfil de varón más capacitado que la mujer para el uso de Internet y que valora más la formación recibida en los diferentes contenidos de la red, con especial incidencia en los servicios más especializados, lo que es coherente con ese mayor nivel de competencia que se atribuye. Las alumnas, por su parte circunscriben el apoyo recibido al ámbito familiar de referencia.

\subsection{Actitudes}

\subsubsection{Resultados del análisis de varianza}

Los resultados se recogen en la Tabla 9[8]. En este caso, son superiores las medias de los chicos en casi todos los ítems valorativos de tipo general (ítem 1-5), siendo las diferencias significativas en ítems que eluden el componente de valoración crítica: ítem 1 ("el uso de Internet es algo imparable que no se puede discutir") y 2 ("Internet aporta mejoras a la sociedad"), lo que posiblemente implica que las chicas son más críticas que los chicos en lo relativo a las repercusiones no tan favorables de la globalización y dela sociedad de la información -no tienen tan claras sus bondades sin matices -. En el mismo sentido, la media 
del ítem 4 ("creo que nos quieren imponer Internet cueste lo que cueste"), es superior en las chicas, aunque la diferencia no es significativa.

Por otra parte, en el ítem 8, relativo a la necesidad del uso de Internet en el centro educativo ("el uso de Internet no es necesario en el centro"), son las chicas las que están más en contra de esta afirmación, lo que implica que valoran más que los chicos la necesidad de usar Internet en el centro (la diferencia de medias es significativa).

Además, en los ítems que reflejan algún tipo de compromiso personal, intención o deseo explícito, (ítems 9-12), en que las puntuaciones son altas en los dos géneros, las medias son superiores en las chicas, dándose diferencia significativa en el ítem 9 ("estoy dispuesto/a a recibir la formación necesaria para el uso de Internet") y en el 12 ("tengo intención de colaborar en proyectos con otros centros que usen Internet"). Todo ello traduce un fuerte compromiso de las chicas -superior al de los chicos, en que también es importante- con el fenómeno Internet, a pesar de las deficiencias en uso y formación, lo que es una base excelente para desarrollar planes de integración.

Tabla 9.-Diferencias en actitudes entre chicos y chicas

\begin{tabular}{|c|c|c|c|c|c|c|}
\hline fiems relation os aCTITUDES frente aluso de Trtemet. & $\begin{array}{c}\text { Medin } \\
\text { Hombre }\end{array}$ & $\begin{array}{c}\text { D. } \\
\text { Tímica }\end{array}$ & $\begin{array}{l}\text { Mfedin } \\
\text { Iffujer }\end{array}$ & $\begin{array}{c}\text { D. } \\
\text { Típica }\end{array}$ & $\begin{array}{c}\text { F. } \\
\text { Anota }\end{array}$ & $\begin{array}{l}\text { Sigifica } \\
\text { ción }\end{array}$ \\
\hline 1.-Eluso de Irtemet es algo imparable, que no se puede diconim & 364 & 130 & 336 & 1,19 & 2721 & 000 \\
\hline 2.-Frtemint oporta mejors a la sociedad & 3,87 & 109 & 3,75 & 1,04 & 733 & 007 \\
\hline 3.-Internat cambiarn las relaciones entre los personx & 337 & 1,28 & 329 & 1,23 & 2,54 & 111 \\
\hline 4.- Creo que nos quieren imponer Irternut auste lo que cueste & 2,80 & 1,35 & 2,83 & 1,28 & 256 & 613 \\
\hline 5. A Irternut sólo accede el que tiene dinero & 2,22 & 134 & 2,14 & 1,27 & 2,11 &, 146 \\
\hline 6-Acceder a Irtemuat y dominarlo me parece dificily yo lo consesuiné & 199 & 1,29 & 190 & 1,18 & 2,44 & 118 \\
\hline $\begin{array}{l}\text { 7. Los jóm enes están cada vex mejor preparados que los adultos para el } \\
\text { acceso a Internet y para su utilimaín }\end{array}$ & 3,88 & 1,28 & 295 & 1,17 & 2,11 & 146 \\
\hline 8.-Estimo que eluso de Internat no es necesario en el centro educationo & 2,13 & 1,28 & 198 & 1,20 & 8,14 & 004 \\
\hline $\begin{array}{l}\text { 9. Estoy diqpuesto/a para recibir la form ación necesaria para eluso de } \\
\text { Intemut. }\end{array}$ & 4,01 & 1,21 & 4,18 & 1,08 & 12,46 & 000 \\
\hline 10.-Deseo unilionr Internet en las closes & 4,23 & 1,17 & 4,24 & 1,09 & 11 & .740 \\
\hline $\begin{array}{l}\text { 11. Tergo interuión de colborar en proyectos del centro que utilicen } \\
\text { Internet. }\end{array}$ & 3,54 & 1,29 & 3,59 & 1,17 & 999 & 318 \\
\hline $\begin{array}{l}\text { 12. -Tenzo irteruión de coliborar en proyectos con otros centros que usen } \\
\text { Internet. }\end{array}$ & 3,08 & 134 & 324 & 1,27 & 907 & 003 \\
\hline
\end{tabular}

\subsubsection{Resultados del análisis discriminante}

El nivel de significación obtenido en la prueba M de Box (0.000) nos indica que no se cumple el supuesto de homogeneidad de las varianzas, por lo que los resultados obtenidos deberemos analizarlos prudentemente.

La función discriminante obtenida presenta una baja correlación canónica $(, 188)$ y un nivel de significación en la prueba Lambda de Wilks de 0.000 .

La estructura de la función discriminante es claramente bipolar, vinculándose a la misma tres ítems en el polo positivo y tres en el negativo (ver tabla 10). Los ítems del polo positivo se refieren principalmente a la valoración sobre la integración de Internet en la sociedad y en el proceso educativo, siendo el ítem que presenta una mayor aportación a la función discriminante el que hace referencia al proceso imparable que a nivel social se está produciendo con la integración de Internet (,782); los otros ítems con aportaciones claramente inferiores se centran en las mejoras que aporta Internet a la sociedad y en la no necesidad de utilizar Internet en los centros educativos. Serían por tanto ítems que harían referencia a una perspectiva crítica sobre la integración de Internet, específicamente en el ámbito educativo. Los ítems del polo negativo, por el contrario, hacen referencia a una mayor implicación y compromiso del alumno en la integración de Internet, siendo el ítem que mayor aportación realiza el relativo a la disposición a recibir formación sobre Internet $(-, 534)$, seguido de la intención de colaborar en proyectos con otros centros que usen Internet y de la valoración de que los jóvenes están mejor preparados que los adultos en la utilización de este recurso.

Tabla 10.-Puntuaciones en la matriz de estructura y coeficientes estandarizados 


\begin{tabular}{|l|c|c|}
\hline & $\begin{array}{c}\text { Matriz de } \\
\text { estructura (a) }\end{array}$ & $\begin{array}{c}\text { Coeficientes } \\
\text { Estandarizados }\end{array}$ \\
\hline "El uso de Intemet es algo "imparable" que no se puede discutir". &, 570 &, 782 \\
\hline "Estoy dispuesto a recibir la formación necesaria para el uso de Intemet". &,- 388 &,- 534 \\
\hline "Tengo intención de colaborar en proyectos con otros centros que usen Internet". &,- 343 &,- 389 \\
\hline "Intemet aporta mejoras a la sociedad". &, 264 &, 344 \\
\hline "Estimo que el uso de Intemet no es necesario en el centro educativo". &, 257 &, 265 \\
\hline $\begin{array}{l}\text { "Los jóvenes cada vez están mejor preparados que los adultos en el acceso y } \\
\text { utilización de Intemet". }\end{array}$ &,- 194 &,- 285 \\
\hline
\end{tabular}

(a) Conelaciones irtragupo combinadas entre las variables discriminantes y las fimciones discriminantes camónicas tịificadas. Variables ordenadas por el tamaño de la correlación con la finción.

Tabla 11.-Funciones en los centroides de los grupos

\begin{tabular}{|l|c|}
\hline \multicolumn{2}{|c|}{ Función } \\
\hline Chico &, 196 \\
Chica &,- 188 \\
\hline
\end{tabular}

Los resultados obtenidos en las funciones discriminantes en las medias de los grupos (ver tabla 12), evidencian que los chicos se sitúan en el polo positivo del factor mientras que las chicas lo hacen en el polo negativo, si bien existe un fuerte solapamiento entre ambos grupos dados los bajos valores obtenidos en ambas funciones. Este solapamiento lleva a obtener un bajo nivel de sujetos clasificados correctamente por los resultados obtenidos en la función discriminante $(58,6 \%)$-ver tabla 12-, encontrándose que los niveles de clasificación correcta son similares en ambos grupos, 58,8\% en el caso de los alumnos y 58,5\% en el de las alumnas.

Tabla 12.-Resultados de la clasificación

\begin{tabular}{|cl|c|c|r|}
\cline { 3 - 4 } \multicolumn{1}{c|}{} & \multicolumn{2}{c|}{$\begin{array}{c}\text { Grupo de pertenencia } \\
\text { pronosticado }\end{array}$} & \multirow{2}{*}{ Total } \\
\hline & Género & Chico & Chica & \\
\hline Original Recuento & Chico & 664 & 466 & 1130 \\
& Chica & 484 & 681 & 1165 \\
& Casos desagrupados & 9 & 7 & 16 \\
\hline$\%$ & Chico & 58,8 & 41,2 & 100,0 \\
& Chica & 41,5 & 58,5 & 100,0 \\
& Casos desagrupados & 57,2 & 42,8 & 100,0 \\
\hline \multicolumn{2}{|c|}{ Clasificados correctamente el $58,6 \%$ de los casos agrupados originales. } \\
\hline
\end{tabular}

Podemos concluir, atendiendo a los resultados obtenidos, que la función discriminante no es lo suficientemente potente para discriminar a los alumnos en relación a la actitud que presentan hacia Internet en función de su género. No obstante, parece existir una cierta tendencia de los chicos a creer en que el uso de Internet a nivel social es algo imparable, que tiene consecuencias positivas pero no en el ámbito educativo en donde su utilización no es necesaria, mientras que las chicas apuntan una mayor implicación y compromiso para formarse en este recurso y su disponibilidad en la participación en proyectos con otros centros.

\subsection{Diferencias en valoración de repercusiones del uso de Internet en la calidad de la educación}

\subsubsection{Resultados del análisis de varianza}

En la Tabla 13 se recogen los resultados[9]. Se trata, en todos los casos de puntuaciones medias bastante altas, que traducen una valoración positiva de ambos géneros de las repercusiones de Internet en la calidad de la educación. Esta valoración positiva es todavía más alta en las chicas que en los chicos, siendo sus medias superiores en todos los ítems, excepto en el ítem 5 ("el uso de Internet me ayuda a sacar mejores notas"). Además, la diferencia es significativa a favor de las chicas en cuatro de los ocho ítems (1: "me ayuda en mis actividades escolares", 2: "sirve de ayuda a algunos de mis compañeros", 6: "me permite conocer a personas distantes y colaborar con ellas", y 7: "creo que lo voy a necesitar haga lo que haga en el futuro"). 
Tabla 13.-Diferencias en valoración de repercusiones en la calidad entre chicos y chicas

\begin{tabular}{|c|c|c|c|c|c|c|}
\hline 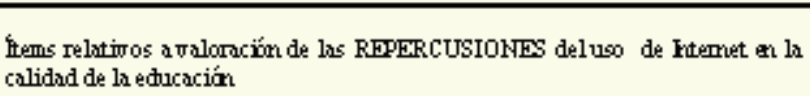 & $\begin{array}{c}\text { Mredin } \\
\text { Hombre }\end{array}$ & $\begin{array}{c}\mathrm{D} \\
\text { T'mica }\end{array}$ & $\begin{array}{l}\text { Mredin } \\
\text { Ifojer }\end{array}$ & $\begin{array}{c}\text { D. } \\
\text { T'mica }\end{array}$ & $\begin{array}{c}\text { F. } \\
\text { AnTơ a }\end{array}$ & $\begin{array}{l}\text { Sigrnfica } \\
\text { ción }\end{array}$ \\
\hline 1.-Eluso de Irtemet me ayuda en mis actio idades escolares & 3,38 & 1,40 & 3,53 & $\overline{1,28}$ & 4,69 & 030 \\
\hline 2.Eluso ... sinte de syuda a algunos de mis comparieros & 3,66 & 1,14 & 3,79 & 105 & 7,65 & 006 \\
\hline 3.Eluso ... me sorta mós medios y recursos para quender & 3,80 & 1,25 & 3,88 & 1,13 & 2,41 & 120 \\
\hline 4.Eluso ... me motimaparatrabaja & 3,14 & 1,33 & 324 & 1,23 & 334 & 068 \\
\hline 5.Eluso ... me ayuda a sacar mejores notas & 299 & 135 & 2,89 & 1,29 & 337 & 068 \\
\hline 6.-Eluso ... me permite conocer a personas distartes y colaborar con ellos & 3,75 & 1,31 & 396 & 1,15 & 16,48 & 000 \\
\hline 7.-Creo que lo toy a necesitar haga lo que haga en elfinmo & 3,82 & 131 & 400 & 1,15 & 11,14 & $\omega 1$ \\
\hline 8-Irtemuet.me gusta mós parn trabajar en clase & 3,66 & 1,36 & 3,68 & 130 & 21 & 640 \\
\hline
\end{tabular}

\subsubsection{Resultados del análisis discriminante}

Se obtiene una función altamente significativa (Lambda de Wilks $\mathrm{p}<0.000$ ). No obstante, el nivel de incumplimiento del supuesto de homogeneidad de las varianzas debe conducirnos a la interpretación de los resultados con cautela ( $\mathrm{M}$ de Box $\mathrm{p}<0.000$ ), especialmente las contribuciones menos relevantes.

A partir de los coeficientes estandarizados y las correlaciones podemos caracterizar el sentido de la función discriminante obtenida -ver tabla 14-.

El polo positivo de la función discriminante se caracteriza por la valoración de que Internet permite conocer y colaborar con personas no importa donde se encuentren, y por la percepción de que motiva a trabajar.

El polo negativo de la función se define solamente con una cuestión: ayuda a obtener mejores notas.

Tabla 14.-Puntuaciones en la matriz de estructura y coeficientes estandarizados

\begin{tabular}{|l|c|c|}
\hline & Matriz de estructura (a) & $\begin{array}{c}\text { Coeficientes } \\
\text { estandarizados }\end{array}$ \\
\hline $\begin{array}{l}\text { El uso de Intemet "me perrite conocer a personas distantes y colaborar } \\
\text { con ellas". }\end{array}$ &, 679 &, 789 \\
\hline El uso de Intemet "me ayuda a sacar mejores notas". &,- 338 &,- 922 \\
\hline El uso de Intemet "me motiva para trabajar". &, 300 &, 506 \\
\hline
\end{tabular}

(a) Conelaciones irtragupo combinadas entre las variables discriminantes y las fimciones discriminantes camónicas tíificadas.

Variables ordenadas por el tamaño de la correlación con la fimción.

Tabla 15.-Funciones en los centroides de los grupos

\begin{tabular}{|l|c|}
\hline & Función \\
\hline Chico &,- 132 \\
Chica &, 127 \\
\hline
\end{tabular}

Los valores de los centroides de ambos grupos (tabla 15) nos indican que las chicas se vinculan al polo positivo de la función discriminante y los chicos al polo negativo, muy moderadamente en ambos casos. Podríamos, pues afirmar que los chicos muestran una visión más pragmática y centrada en las repercusiones en las calificaciones y que las chicas se vinculan a aspectos relacionados con la motivación por el trabajo, junto a una percepción de un elemento especialmente característico de estas tecnologías: la comunicación potenciada con otras personas.

Tabla 16.-Resultados de la clasificación 


\begin{tabular}{|cl|c|c|r|}
\cline { 3 - 4 } \multicolumn{1}{c|}{} & \multicolumn{2}{c|}{$\begin{array}{c}\text { Grupo de pertenencia } \\
\text { pronosticado }\end{array}$} & \multirow{2}{*}{ Total } \\
\hline & Género & Chico & Chica & \\
\hline Original Recuento & Chico & 661 & 469 & 1130 \\
& Chica & 582 & 583 & 1165 \\
& Casos desagrupados & 4 & 11 & 16 \\
\hline$\%$ & Chico & 58,5 & 41,5 & 100,0 \\
& Chica & 49,9 & 50,1 & 100,0 \\
& Casos desagrupados & 27,7 & 72,3 & 100,0 \\
\hline \multicolumn{2}{|c|}{ Clasificados correctamente el $54,2 \%$ de los casos agrupados originales. } \\
\hline
\end{tabular}

Finalmente, la validación del modelo discriminante permite un nivel pobre de buena identificación -ver tabla 16- que se acerca al $60 \%$ en el caso de los chicos. Las chicas tienen una discriminación más pobre a partir del modelo, un $50 \%$ de los casos que coincide con la probabilidad de identificación por azar. Podemos, pues, afirmar que el modelo no resulta satisfactorio para una identificación de diferencias consistentes entre chicos y chicas, no obstante el alto nivel de significación obtenido.

\subsection{Resultados del análisis discriminante de los ítems significativos de los cuatro bloques temáticos.}

De cara a llevar a cabo una reintegración de los datos obtenidos se ha realizado un análisis discriminante paso a paso sobre las variables significativas seleccionadas a partir de los análisis discriminantes anteriores en los cuatro ámbitos considerados (Uso, Formación, Actitudes y Repercusiones en la Calidad), lo que permitirá adquirir una perspectiva global de las diferencias entre géneros. Se obtiene una función altamente significativa (Lambda de Wilks $\mathrm{p}<0.000$ ). No obstante, el nivel de incumplimiento del supuesto de homogeneidad de las varianzas debe conducirnos a la interpretación de los resultados con cautela (M de Box $\mathrm{p}<0.000$ ), especialmente las contribuciones menos relevantes. A partir de los coeficientes estandarizados y las correlaciones podemos caracterizar el sentido de la función discriminante obtenida.ver Tabla 17 de coeficientes estandarizados y correlaciones intragrupo-.

El polo positivo de la función discriminante se caracteriza por las horas que el sujeto dedica en casa a jugar con el ordenador y el nivel de conocimiento y utilización de Internet que posee. Secundariamente existen diferentes aspectos que contribuyen a matizar el sentido de esta polaridad positiva: la utilización de Internet para usos "minoritarios" -más allá de los servicios fundamentales recogidos en otras alternativas-, la consideración de que el fenómeno de Internet es algo imparable y aporta mejoras a la sociedad, la valoración de que Internet le ayuda a obtener mejores notas, y la apreciación de que Internet no es necesaria en el centro educativo. Tenemos pues, como elementos básicos ítems vinculados al uso y conocimiento, matizados por algunas cuestiones relativas a aspectos actitudinales y de repercusiones en la calidad educativa.

El polo negativo de la función se define con mucha menor consistencia, dado que tanto sus contribuciones a la función como sus correlaciones con la misma son menores. Los elementos más poderosos son los relativos a uso de Internet: la utilización fuera de centro de charlas (chats), y la menor intensidad de uso del ordenador en su domicilio para tareas que no implican el juego. Secundariamente aparecen diversos matices relacionados con elementos actitudinales y de repercusiones en la calidad educativa: disponibilidad para recibir la formación precisa para utilizar Internet, la consideración de que utilizar Internet le motiva a trabajar y le permite conocer a personas distantes y colaborar con ellas.

Tabla 17.-Puntuaciones en la matriz de estructura y coeficientes estandarizados 


\begin{tabular}{|c|c|c|}
\hline & $\begin{array}{l}\text { Matriz de } \\
\text { estructura (a) }\end{array}$ & $\begin{array}{l}\text { Coeficientes } \\
\text { Estandarizados }\end{array}$ \\
\hline ¿Cuántas horas semanales dedic as en casa a usar el ordenador para "jugar"? &, 548 & 613 \\
\hline ¿Cómo calificarías tu nivel de conocimiento y manejo de Intemet? & 474 & ,433 \\
\hline $\begin{array}{l}\text { Indica la frecuencia de uso mediante Intemet fuera del centro para otros } \\
\text { (especificar) }\end{array}$ &, 389 & ,348 \\
\hline "El uso de Intemet es algo "imparable" que no se puede discutir". &, 307 & ,304 \\
\hline "Estimo que el uso de Internet no es necesario en el centro educativo". & 238 & 223 \\
\hline "Intemet aporta mejoras a la sociedad". & 222 & 220 \\
\hline "Estoy dispuesto a recbir la formación necesaria para el uso de Intemet". &,- 160 &,- 277 \\
\hline El uso de Intemet "me ayuda a sacar mejores notas". & 145 & 310 \\
\hline $\begin{array}{l}\text { El uso de Internet "me perrmite conocer a personas distantes y colaborar con } \\
\text { ellas". }\end{array}$ &,- 122 &,- 250 \\
\hline El uso de Internet "me motiva para trabajar". &,- 045 &,- 250 \\
\hline $\begin{array}{l}\text { ¿Cuántas horas semanales dedic as en casa a usar el ordenador para otras cosas - } \\
\text { sin jugar-? }\end{array}$ &, 021 &,- 336 \\
\hline $\begin{array}{l}\text { Indica la frecuencia de uso mediante Intemet fuera del centro para charlas (chats, } \\
\text { IRC, etc.) }\end{array}$ &,- 011 & -.336 \\
\hline
\end{tabular}

(a) Correlaciones intra-gupo oombinadas entre las variables discriminantes y las funciomes discrininartes canónicas tịificadas Variables ordenadas por el tamaño de la correlación con la fimción.

Tabla 18.-Funciones en los centroides de los grupos

\begin{tabular}{|c|c|}
\hline \multicolumn{2}{|c|}{ Función } \\
\hline Chico & 440 \\
Chica &,- 467 \\
\hline
\end{tabular}

Los valores de los centroides de ambos grupos nos indican que los chicos se vinculan al polo positivo de la función discriminante y las chicas al polo negativo. A la vista de los resultados podríamos, pues afirmar que:

- Los chicos juegan mucho más con el ordenador y el nivel de conocimiento de Internet es claramente superior al de las chicas. También hacen algunas cosas en Internet, relativamente específicas -como la utilización de las herramientas para redes "peer to peer", para música , video,...-. Las chicas, en cambio, utilizan con mayor frecuencia las charlas electrónicas y, adicionalmente, presentan menor nivel de actividad con el ordenador en su domicilio en cuestiones que no tienen que ver con jugar. En conjunto, tanto el conocimiento como el uso del ordenador -en términos generales y por lo que se refiere al acceso a Internet- es claramente superior en los chicos que en las chicas.

- Por lo que se refiere a los aspectos actitudinales y de repercusiones en la calidad educativa, los chicos consideran en mayor medida que Internet es un fenómeno imparable, que les ayuda a sacar mejores notas y que aporta mejoras a la sociedad. No obstante, también piensan que Internet no es necesario en el centro educativo lo que contradice en parte su posicionamiento frente a Internet, y nos demuestra cierta heterogeneidad en las posiciones de los chicos. Además, en términos globales esta cuestión muestra que los chicos presentan una relación con Internet predominantemente vinculada a aspectos personales, solamente de forma tangencial relacionada con el hecho educativo. Por su parte, las chicas manifiestan actitudes que implican su motivación para mejorar sus conocimientos sobre Internet, su percepción de que Internet las motiva a trabajar y les permite conocer personas distantes.

Tabla 19.-Resultados de la clasificación 


\begin{tabular}{|cl|c|c|r|}
\cline { 3 - 4 } \multicolumn{1}{c|}{} & \multicolumn{2}{c|}{$\begin{array}{c}\text { Grupo de pertenencia } \\
\text { pronosticado }\end{array}$} & \multirow{2}{*}{ Total } \\
\hline & Género & Chico & Chica & \\
\hline Original Recuento & Chico & 711 & 419 & 1130 \\
& Chica & 352 & 813 & 1165 \\
& Casos desagrupados & 7 & 9 & 16 \\
\hline$\%$ & 63,0 & 37,0 & 100,0 \\
& Chico & 30,2 & 69,8 & 100,0 \\
& Chica & 42,6 & 57,4 & 100,0 \\
\hline \multicolumn{2}{|c|}{ Casos desagrupados } & \multicolumn{3}{c}{} \\
\hline \multicolumn{2}{|c|}{ Clasificados correctamente el $66,4 \%$ de los casos agrupados originales. } \\
\hline
\end{tabular}

Finalmente, la validación del modelo discriminante permite un nivel de buena identificación satisfactorio ver Tabla 19-. En ambos casos se supera el 60\% de identificaciones correctas de los individuos pertenecientes a cada grupo, alcanzándose en promedio la identificación de dos tercios de los casos. Las chicas tienen una discriminación más precisa a partir del modelo, casi un 70\% de los casos. Así, resulta notable que aunque la definición del polo negativo de la función discriminante es más pobre menos extremada o apartada de cero-, esta resulta bastante más precisa como perfil diferenciador que en el caso de los chicos.

\section{4.- Conclusiones y recomendaciones}

Los datos del análisis univariado de diferencias hacen patente una cierta "brecha digital" entre chicos y chicas de Educación Secundaria Obligatoria de la Comunidad Valenciana favoreciendo las diferencias a los chicos en disponibilidad de conexión en casa, en uso de los diferentes servicios de Internet y en formación, con un mayor nivel de competencia por parte de los varones en el manejo de Internet tanto en servicios generales como en servicios más especializados. Sin embargo, las actitudes hacia la integración de las TIC de las chicas son todavía más positivas que las de los chicos -que también lo son- y reflejan un fuerte compromiso con el fenómeno y la intención de formarse y de cooperar en proyectos de uso. Si a ello unimos que valoran muy positivamente las repercusiones de Internet sobre la calidad de la educación, sobre el aprendizaje y el propio trabajo -también en un nivel más alto que los chicos-, podemos concluir que esa brecha se puede cerrar con relativa facilidad, siempre que las políticas de integración se hagan con conciencia de ello y que los agentes que deben llevarla a cabo -especialistas, profesores, directivos de los centros, familias...- trabajen para compensar esas diferencias que se dan a favor de los chicos.

Los datos del análisis multivariado, complementarios de los anteriores, reflejan que las diferencias debidas al género se centran fundamentalmente en cuestiones relativas al conocimiento y manejo de Internet, secundariamente complementadas con algunos aspectos actitudinales y de percepción sobre sus repercusiones en la calidad educativa, lo que es consistente con los datos aportados por la perspectiva univariada. Efectivamente, en un momento en el que el propio acceso a Internet y los conocimientos sobre sus herramientas y posibilidades es bastante reducido, es coherente que estos elementos se sitúen en un plano central para definir el problema de las diferencias entre chicos y chicas.

Resulta particularmente notable que la diferencia más poderosa respecto al uso de Internet se da, para el caso de los chicos, en la utilización de "otros usos-servicios" que hemos denominado como minoritarios. Así, aunque los chicos mantienen un nivel de competencia y de uso del ordenador y de las herramientas relacionadas con Internet claramente superior a las chicas, estas opciones "atípicas" sigue siendo una fuente principal para explicarlas frente a otras muchas posibilidades. De hecho, la presencia y nivel de implicación de los chicos en los juegos en Internet e intranet -en cibercafés, domicilios,...-, los accesos mediante redes "peer to peer" a contenidos musicales y de vídeo, etc. alcanza una extensión e intensidad mucho mayor. Este es un fenómeno que ha comenzado a estudiarse a un nivel muy primario hasta la actualidad y que tiene raíces muy profundas -p.e. existen ya algunos indicios respecto a los patrones de género de los juegos que parecen condicionar el acceso e implicación de las chicas- que se aprecian desde el acercamiento al ordenador, en particular, y a Internet, en general, que muestran ambos colectivos. En términos más globales, sigue siendo muy superior la presencia de chicos en los estudios -de cualquier nivel- relacionados con estas tecnologías y estas diferencias aparecen asimismo en un plano mucho más general -la proporción de chicas en actividades no formales relacionadas con estas tecnologías-como los "campus" que han ido proliferando- es abrumadoramente minoritaria. 
En otro orden de cosas, los elementos relativos a las actitudes y percepciones respecto a Internet, no obstante su menor peso en la explicación de diferencias, resultan particularmente significativos. Así, los chicos tienden a expresar ideas que se mueven entre lo pragmático y la idealización de Internet. Por su parte, las chicas manifiestan predisposiciones a cubrir las importantes carencias que poseen y su motivación a trabajar más con esta herramienta, junto a tendencias mucho más ajustadas a algunos de los elementos inherentes a este soporte: la colaboración y comunicación con otras personas. También resulta relevante la excesiva vinculación de la utilización de Internet por los chicos a un plano personal -en buena parte lúdico-, que llega a condicionar posiciones ciertamente contradictorias que se reflejan en afirmaciones que señalan la no pertinencia de Internet en el ámbito educativo.

Aludíamos en el primer párrafo de este apartado a una cierta "brecha digital". Hay que tener presente que esa "brecha" presenta una evidente complejidad -como es habitual en el análisis de las misma en otros ámbitos educativos y sociales-, mostrándose múltiples dimensiones relevantes (OCDE, 2001). Así, el entorno social general y, muy particularmente, el familiar deberán atenderse para acortar las diferencias existentes -disponibilidad, utilización,...- entre chicos y chicas en igualdad de contextos sociofamiliares. De igual forma, deberá atenderse el propio ámbito educativo formal, teóricamente igual para todos, en el que siguen evidenciándose claras diferencias al respecto. Por tanto, cualquier enfoque respecto a este problema deberá tomar en consideración los múltiples planos y dimensiones que le afectan y que no son particularmente diferentes de los que se encuentran en el estudio de la problemática del género en su conjunto. Las propias actitudes y predisposiciones al respecto que manifiestan las chicas pueden constituir una sólida base en que apoyarse para encarar con éxito esta problemática.

La cuestión es, pues, que en una de las expresiones básicas de algo tan nuevo como la Sociedad de la Información vuelven a reproducirse las características sociales y culturales imperantes en las etapas anteriores. El riesgo añadido es, obviamente, que estas nuevas circunstancias pueden perpetuar o incrementar estas diferencias si no se toman en consideración y se actúa de forma explícita al respecto. Existen algunos indicios de que los planes de actuación decidida al respecto pueden cambiar de forma bastante radical esta situación en un plazo relativamente corto, particularmente si se utilizan las herramientas de educación formal (Rhode y Shapiro, 2000).

\section{Bibliografía}

CASTELLS, M. (2000) La era de la información. Vol. I. La sociedad red. Madrid, Alianza.

GARGALlO, B., SUÁREZ, J., MORANT, F., MARÍN, J.M., MARTÍNEZ, M. y DÍAZ, I. (2001)Un primer diagnóstico del uso de Internet en los centros escolares de la Comunidad Valenciana. Procesos de formación y efectos sobre la calidad de la educación. Informe de investigación. Manuscrito no publicado.

GRUPO WEB DE MS (2001) Tendiendo un puente sobre la divisoria digital. Documento electrónico http://www.ms-an.dk/uk/Politics_press/ Policy_papers/ictspanish.htm

HAIR, J.F., ANDERSON, R.E., TATHAM, R.L. y BLACK, W.C. (1999) Análisis Multivariante. (5ª ed.). Madrid, Prentice Hall.

KERREY, R. (2000) The power of Internet for learning. Moving from Promise to Practice. Report of the web-based Education Comission to the President of the Congress of the United States. Documento electrónico http://interact.hpcnet.org/webcomission/index.htm

MARINA, J.A. (1999) El timo de la sociedad de la información. En MARINA, J.A., JOYANES, L., TOHARIA, M., BARTOLOMÉ, A.R. y MARTÍN, E. Educación e Internet. Documentos del $1^{\circ}$ Congreso Educación e Internet. Educnet 99. Madrid, Santillana, 13-19.

OCDE (2001) Understanding digital divide. Paris, OCDE (http://www.oecd.org)

PRICE, L. (2000) Lessons on Internet Access and Use Statistics. The Digital Divide: Enhancing Access to ICTs. Paris, OCDE Workshop.

RHODE, G.I. y SHAPIRO, R. (2000) Falling through the Net: Towards Digital Inclusion. Report NTIAESA. Washington DC, US Department of Comerce. 
SHASSHAANI, L. y KHALILI, A. (2001) Gender and computers: similarities and differences in Iranian collage students' attitudes towards computers. Computers \& Education, 37, 363-375.

SINGLETON, S. y MAST, C. (2000) How Does the Empty Glass Fill?. A Modern Philosophy of the Digital Divide. EDUCAUSE. Documento electrónico http://www.educause.edu/asp/doclib/abstract.asp? ID=ERM0062

STEVENS, J. (1996) Applied Multivariate Statistics for the Social Sciences. (3rd. ed.). Mahwah, NJ.LEA.

SUÁREZ, J., GARGALLO, B., TORRECILLA, M., MARÍN, J.M., MORANT, F. y DÍAZ, I. (2001) La "División Digital" en el proceso de integración de las TIC en la Educación. Diferencias de género entre profesores de E.S.O. de la Comunidad Valenciana. Ponencia presentada en la Conferencia Internacional sobre Educación, Formación y Nuevas Tecnologías Virtual Educa 2002. Valencia, 12-14 de junio. Documento electrónico http://www.virtualeduca.org/virtual/html/princip17.htm

\section{Notas}

[1] Una primera versión de este trabajo se presentó en el XXI Seminario Interuniversitario de Teoría de la Educación celebrado en Granada en noviembre de 2002.

[2] Se trata de una investigación desarrollada a lo largo de los años 2000-2001 bajo el título "Un primer diagnóstico del uso de Internet en los centros escolares de la Comunidad Valenciana. Procesos de formación y efectos sobre la calidad de la educación", subvencionada por el Instituto Valenciano de Evaluación y Calidad Educativa, llevada a cabo por un equipo dirigido por el primer firmante de este trabajo.

[3] En las tablas se recogen descriptivos básicos de cada variable, F de Anova y su significación.

[4] Excepto en el primer ítem, en que la respuesta posible es dicotómica, hay cinco grados de respuesta a los ítems del cuestionario de los que reflejamos los resultados, que van del 1 al 5. Para los ítems 2-10 (horas semanales dedicadas...) las cinco respuestas posibles son 1: ninguna; 2: menos de 5; 3: entre 5 y 10; 4: entre 10 y 20; y 5: más de 20. Para los ítems 11-47 (frecuencia de uso...), las respuestas posibles son 1: nunca, 2: menos de una vez al mes; 3: al menos una vez al mes; 4: al menos una vez por semana; y 5: diariamente. Las medias obtenidas son siempre muy bajas, lo que implica necesidades básicas de dotación -hay todavía algún centro sin ninguna conexión a Internet, otros con conexiones insuficientes, y bastantes alumnos sin disponibilidad de conexión en casa-.

[5] Aunque hay que tener presente el signo negativo de su relación con la función discriminante, que indica menos horas de dedicación a estas tareas, el valor es tan reducido que prácticamente se sitúa en el punto neutro de la misma.

[6] Encontramos resultados similares analizando diferencias de género en el uso de las TIC en los profesores/as de la muestra de la investigación (Suárez, Gargallo, Torrecilla, Marín, Morant y Díaz, 2002). Se apreciaron diferencias significativas a favor de los profesores en la gran mayoría de cuestiones relacionadas con la utilización de Internet tanto en el centro como en su domicilio, a nivel personal y en el trabajo curricular con los alumnos. Podría argüirse en este caso que se trata de otra generación; sin embargo, encontramos que el perfil se repite con sus alumnos, lo que no deja de ser preocupante.

[7] La escala de valoración de los diferentes ítems es también de 5 grados, siendo en el ítem 1 las respuestas posibles 1: No sé manejar Internet; 2: Principiante; 3: Medio; 4: Avanzado; 5: Experto. En los ítems 2-8 y 10-16, las posibles respuestas son 1: Nada; 2: Poco; 3: Regular; 4: Bastante; 5: Mucho. En el ítem 9 las posibles respuestas son 1: No he recibido formación; 2: Un curso o cursos de hasta 50 horas; 3: Un curso o cursos de entre 50 y 100 horas; 4: Un curso o cursos de entre 100 y 200 horas; 5: un curso o cursos de más de 200 horas. En los ítems 17-26, las posibles respuestas son 1: Nula; 2: Insuficiente; 3 : Suficiente; 4: Buena; 5: Excelente. Como en el bloque anterior, las medias obtenidas son casi siempre muy bajas. 
[8] Se utilizó una escala de puntuación de 5 grados, siendo 1: Totalmente en desacuerdo; 2: Parcialmente en desacuerdo; 3: Indiferente; 4: Parcialmente de acuerdo; y 5: Totalmente de acuerdo. Obsérvese que en este bloque las puntuaciones medias otorgadas son sustancialmente más altas que en los anteriores.

[9] Se utilizó la misma escala de puntuación que en el bloque anterior. También en este caso las puntuaciones son significativamente más altas que en los dos primeros bloques.

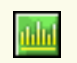

(C) Ediciones Universidad de Salamanca Páginas mantenidas por Francisco Ignacio Revuelta Domínguez y Luis González Rodero

webmrte@usal.es 